\title{
Gene synthesizing, expression and immunogenicity characterization of recombinant translation elongation factor 2 from Dermatophagoides farinae
}

\author{
DESHENG CHEN ${ }^{1,2^{*}}$, QINGHUI FU ${ }^{1 *}$, JIANLI LIN $^{1 *}$, CHENGSHEN HU $^{1}$, \\ NANA HUANG ${ }^{1}$, KE XIN CHANG $^{1}$, BAOQING SUN ${ }^{2}$ and ZHIGANG LIU ${ }^{1,2}$
}

\begin{abstract}
${ }^{1}$ State Key Laboratory of Respiratory Disease for Allergy at Shenzhen University, Shenzhen Key Laboratory of Allergy and Immunology, Shenzhen University School of Medicine, Shenzhen, Guangdong 518060; ${ }^{2}$ Department of Allergy, Affiliated Luohu Hospital of Shenzhen University, Shenzhen Luohu Hospital Group, Shenzhen, Guangdong 518000, P.R. China
\end{abstract}

Received January 7, 2018; Accepted September 4, 2019

DOI: $10.3892 / \mathrm{mmr} .2019 .10786$

\begin{abstract}
House dust mite (HDM) hypersensitivity increasingly affects millions of individuals worldwide. Although numerous major allergens produced by HDM species have been characterized, some of the less potent allergens remain to be studied. The present study aimed to obtain the recombinant allergen of Translation Elongation Factor 2 (TEF 2) from the HDM Dermatophagoides farinae by synthesizing, and then expressing the recombinant TEF 2 to identify its immunogenicity. In the present study, the D.farinae TEF 2 (Der f TEF 2) was synthesized, expressed and purified. The molecular characteristics of Der f TEF 2 were analyzed using bioinformatics approaches. The recombinant protein was purified via affinity chromatography, and the allergenicity was assessed using immunoblotting, ELISAs and skin prick tests. The gene for TEF 2 consists of 2,535 bp and encodes an 844 -amino acid protein. A positive response to recombinant Der f TEF 2 was detected in $16.2 \%$ of 37 patients with HDM allergies using skin prick tests. In addition, the immunoblotting indicated that the protein showed a high ability to bind serum IgE from patients allergic to HDMs, and that the recombinant TEF 2 was highly immunogenic. Bioinformatics analysis predicted 17 peptides as B cell epitopes (amino acids 29-35, 55-64, 92-99, 173-200, 259-272, 311-318, 360-365, 388-395, $422-428,496-502,512-518,567-572,580-586,602-617$,
\end{abstract}

Correspondence to: Dr Zhigang Liu, State Key Laboratory of Respiratory Disease for Allergy at Shenzhen University, Shenzhen Key Laboratory of Allergy and Immunology, Shenzhen University School of Medicine, Room 722, 3688 Nanhai Blvd, Shenzhen, Guangdong 518060, P.R. China

E-mail: lzg195921@126.com

${ }^{*}$ Contributed equally

Key words: Dermatophagoides farinae, allergen, translation elongation factor, allergenomics, purification
785-790,811-817 and 827-836) and 14 peptides as T cell epitopes (amino acids 1-15, 65-79, 120-134, 144-159, 236-250, 275-289, 404-418, 426-440, 463-477, 510-524, 644-658, 684-698, 716-730 and 816-830). The software DNAStar predicted the secondary structure of TEF 2 , and showed that $27 \alpha$-helices and five $\beta$-sheets were found in the protein. In conclusion, the present study cloned and expressed the Der f TEF 2 gene, and the recombinant protein exhibited immunogenicity, providing a theoretical bases, and references, for the diagnosis and treatment of allergic disease.

\section{Introduction}

There has been a dramatic increase in the prevalence of allergic diseases, which are common and frequently present in clinical practice $(1,2)$. Dermatophagoides pteronyssinus and Dermatophagoides farinae are the major house dust mite (HDM) species, and are the most significant sources of indoor allergens for inhalation, causing allergic diseases, including allergic asthma and allergic rhinitis atopic dermatitis $(3,4)$. Desensitization treatment with HDM extracts is currently one of the most effective treatments against allergies to HDMs (5). However, it is difficult to ensure the consistency of the natural extracts of HDMs because of their complex components, including inflammatory molecules (ceramides, kallikreins and endotoxin), that are usually responsible for side effects and poor efficacy (6). Therefore, recombinant allergens are given prior to their natural counterparts in HDM-specific immunotherapy to improve the efficacy and safety of presentation, diagnosis and clinical immunotherapy (7).

Decades of research have revealed and characterized $>30$ different allergens in D. farinae; group 1 and group 2 allergens are the most clinically relevant, as they possess IgE-binding activity in $>80 \%$ of patients with HDM allergies (8-10). These allergens induce $\mathrm{T}$ helper 2 immune responses by encoding cysteine proteases and epididymal proteins $(9,11)$. Translation elongation factors (TEFs) are expressed in Cladosporium, Rubber and HDM (12-14). The allergenicity of the TEF 2 in D. farinae has not been defined. Therefore, understanding the 
allergenicity of TEF 2 will be important for the detection and treatment of allergic responses.

Currently, with the development of bioinformatics, the concept of precision medicine is emerging. Bioinformatics can predict key target spots of a disease, which can provide critical references for the drug and therapy development $(15,16)$. Previously, epitope peptide vaccines were considered to have excellent potential application prospects, therefore, it is critical to predict the epitope of novel allergens (17-19). Novel vaccines, synthesized based on B-cells of the T-cell epitope, can activate the produce specific antibodies from human $\mathrm{B}$ cells, and can also eliminate infected cells by activating cytotoxic lymphocytes (17). Therefore, the present study cloned, expressed and purified TEF 2, and evaluated its allergenicity. The properties of TEF 2 were predicted by using bioinformatics tools, providing valuable information for further vaccine development.

\section{Materials and methods}

Sera and skin prick test (SPT). The written informed consent was obtained from each participant for the use of peripheral blood samples and SPT. The serum and SPT of 37 allergic patients (22 female, 15 male; 8-86 years) were from The First Affiliated Hospital of Guangzhou Medical University. The sera of 3 healthy subjects (3 male, 8-15 years) were recruited from Shenzhen Children's Hospital. The 11 children among the subjects were approved by legal guardians. The samples were collected between January 2014 and December 2015. the present study was approved by the ethic Committee of the Institutional Review Board of the School of Medicine, Shenzhen University.

Obtaining the gene encoding TEF 2. In a previous study, the draft genome of Dermatophagoides farinae were assembled using high-throughput sequencing (20). The gene sequence of Der $\mathrm{f}$ TEF 2 was obtained by high-throughput sequencing from Dermatophagoides farinae (Hong Kong Bioinformatics Centre, Hong Kong) and then the gene of Der f TEF 2 was synthesizing by Sangon Biotech in Shanghai. The $18 \mu \mathrm{l}$ cDNA mixed with $2 \mu \mathrm{l}$ (1/10 volume) of 10X DNA loading buffer (Beijing Solarbio Science \& Technology Co., Ltd.) were added to the sample tank and the electrophoresis started. After the electrophoresis, the gel was stained by ethidium bromide, and images were captured under a UV lamp with a wavelength of $302 \mathrm{~nm}$.

Expression and purification of recombinant TEF 2. The cDNA of Der f TEF 2 was inserted into a pMD-18T vector (Takara Bio, Inc.), cloned into the BamHI and HindIII sites and the plasmids were heat-transformed into E. coli Top10 (Invitrogen; Thermo Fisher Scientific, Inc. The bacteria were incubated in LB medium (Beijing Solarbio Science \& Technology Co., Ltd.) with $100 \mathrm{mg} / \mathrm{l}$ ampicillin and cultured overnight at $37^{\circ} \mathrm{C}$. The recombinant colonies were transferred into LB medium with ampicillin to expand the culture, followed by plasmid extraction using plasmid DNA extraction kit (Omega Bio-Tek, Inc). The recombinant plasmid was characterized using Bam $\mathrm{HI}$ and sequenced by BGI Group. After sequencing, the correct clone was ligated into the pET-32a expression vector (Novagen; Merck $\mathrm{KGaA}$ ) at $37^{\circ} \mathrm{C}$ for $4 \mathrm{~h}$. Initial cloning was performed with the E. coli strain Top10 cultured overnight at $37^{\circ} \mathrm{C}$; a single colony was picked, and the plasmid was extracted for identification using Bam HI and HindIII restriction enzymes. pET-32a-TEF 2 was transformed into $E$. coli BL21 (Invitrogen; Thermo Fisher Scientific, Inc.) cells for expression. The cells were grown in LB medium supplemented with $50 \mu \mathrm{g} / \mathrm{ml}$ ampicillin until the logarithmic phase was reached (A600 nm =0.6-0.9). Expression of TEF 2 was induced by adding $20 \mu \mathrm{l}$ of isopropyl-D-thiogalactopyranoside (IPTG; $1 \mathrm{~mol} / \mathrm{l}$ ) to the $\mathrm{LB}$ medium. After $4 \mathrm{~h}$ of continued incubation at $37^{\circ} \mathrm{C}$, the bacteria were harvested via centrifugation at $11,180 \mathrm{x} \mathrm{g}$ at room temperature for $5 \mathrm{~min}$ and $1 \mathrm{ml}$ of the bacterial culture resuspended in $100 \mu \mathrm{l}$ deionized water and mixed with 20-25 $\mu \mathrm{l} 10 \mathrm{X}$ protein SDS-PAGE loading buffer (Beijing Solarbio Science \& Technology Co., Ltd.). The expression of the recombinant proteins was analyzed using 12\% SDS-PAGE electrophoresis.

Following the induced expression of the recombinant protein, the samples were lysed using ultrasonic treatment at $4^{\circ} \mathrm{C}$ and an amplitude of $38 \%$ for $5 \mathrm{~min}$ ( $1 \mathrm{sec}$ pulse on and $0.1 \mathrm{sec}$ pulse off). The supernatant was purified using a balanced Ni-NTA column (ShangHai, Sangon Biotech, cat. no. C600792, 5 ml, $90 \mu \mathrm{m})$ at a speed of $2 \mathrm{ml} / \mathrm{min}$ and $4^{\circ} \mathrm{C}$. After washing with a washing buffer $(50 \mathrm{mM}$ Tris, $40 \mathrm{mM}$ imidazole and $0.5 \mathrm{M}$ $\mathrm{NaCl}, \mathrm{pH}$ ), the protein was eluted slowly using an elution buffer (50 mM Tris, 0.3 M imidazole and $0.2 \mathrm{M} \mathrm{NaCl}, \mathrm{pH} 8$ ). The elution peak was collected by elution buffer ( $50 \mathrm{mM}$ Tris, $0.3 \mathrm{M}$ imidazole and 0.2 $\mathrm{M} \mathrm{NaCl}, \mathrm{pH} \mathrm{8}$ ).

ELISAs. The HDMs allergic sera IgE antibodies specific to TEF 2 were measured indirectly using ELISAs. Briefly, 96-well microtiter plates were coated with $100 \mathrm{ng} /$ well of TEF 2 at a concentration of $1 \mu \mathrm{g} / \mathrm{ml}$ in carbonate buffered solution (15 $\mathrm{mM} \mathrm{Na}_{2} \mathrm{CO}_{3}$ and $\left.35 \mathrm{mM} \mathrm{NaHCO} 3, \mathrm{pH} 9.5\right)$ at $4^{\circ} \mathrm{C}$ overnight and then blocked with $200 \mu 13 \% \mathrm{BSA}$ in PBS at $37^{\circ} \mathrm{C}$ for $120 \mathrm{~min}$. Each well was prepared with the serum (diluted 1:5; $100 \mu \mathrm{l} /$ well) as the primary antibody or 3\% BSA (as a negative control, Beijing Solarbio Science \& Technology Co., Ltd.) and incubated at $37^{\circ} \mathrm{C}$ for $60 \mathrm{~min}$. The plates were incubated with peroxidase-conjugated goat anti-human $\operatorname{IgE}(1: 2,000$, A18793, Invitrogen; Thermo Fisher Scientific, Inc.) at $37^{\circ} \mathrm{C}$ for $60 \mathrm{~min}$. Each incubation step was followed by three washes with PBS-0.05\% Tween 20 (PBST). Color was developed by adding $100 \mu \mathrm{l} / \mathrm{well} \mathrm{TMB}$ at $37^{\circ} \mathrm{C}$ for $10 \mathrm{mins}$ and terminated by the addition of $2 \mathrm{M} \mathrm{H} 2 \mathrm{SO} 4$ (50 $\mu \mathrm{l} /$ well). Absorbance was determined using a microplate reader at $450 \mathrm{~nm}$.

Determination of inhibition using ELISA. The serum of patients allergic to TEF 2 was used for inhibition experiments to determine the cross reactivity among TEF 2 and HDMs. Patients' sera (supplied pre-diluted in 2\% BSA in PBST) were pre-incubated with purified Der f TEF 2 or dust mite extract (DME) obtained from mites that were cryopreserved in liquid nitrogen and then ground to a powder (final concentration: $0.00001,0.0001,0.001,0.01$ or $1 \mathrm{~g} / \mathrm{ml})$ at $4^{\circ} \mathrm{C}$ overnight. Pre-incubated Der f TEF 2 or DME as the primary antibody were added to the microtiter plates pre-coated with TEF 2 or DME $(0.1 \mu \mathrm{g} /$ well $)$ and the ELISA was performed as in the previous section. According to ELISA test procedures, data 


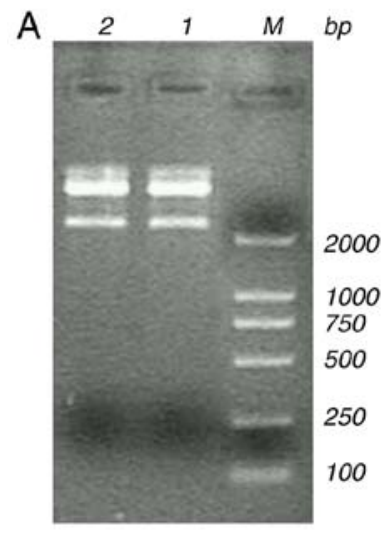

B

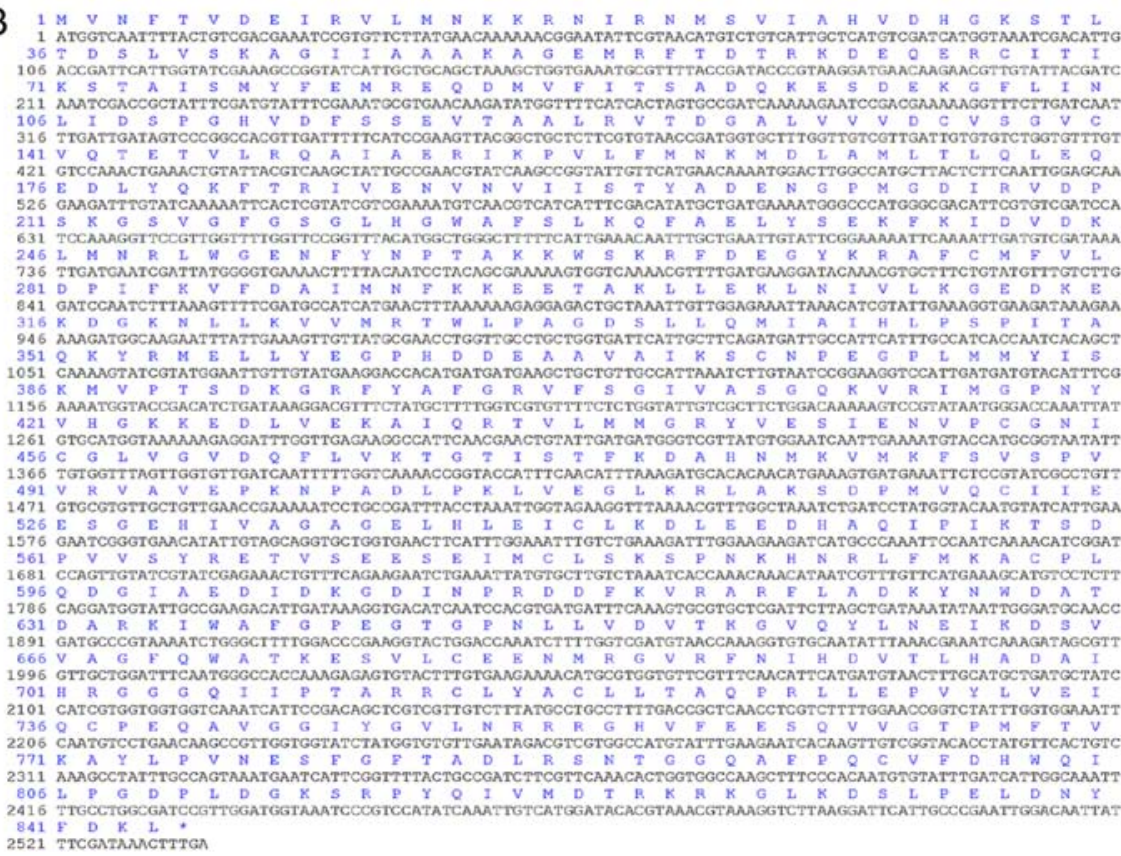

Figure 1. The cDNA of Der $\mathrm{f}$ TEF 2. (A) Agarose gel electrophoresis of products from the pET-32a-Der $\mathrm{f}$ TEF 2 double digest using BamHI and EcoRI. Lanes: M, DNA Marker; 1 and 2, Der f TEF 2 DNA. (B) Amino acid sequences of Der f TEF 2. Der f, Dermatophagoides farinae; TEF 2, translation elongation factor 2.

from three experiments were collected. The inhibition rates were calculated according to the following formula: Inhibition $(\%)=\left(\mathrm{OD}_{0}-\mathrm{OD}_{\text {inhibitor }}\right) /\left(\mathrm{OD}_{0}-\mathrm{OD}_{\mathrm{BSA}}\right)$, where: $\mathrm{OD}_{0}=$ the optical density of antigen without any inhibitor, $\mathrm{OD}_{\text {inhibitor }}=$ the optical density after adding an inhibitor (0.00001, 0.0001, 0.001, 0.01 or $1 \mathrm{~g} / \mathrm{ml}$ TEF 2 or DME), and $\mathrm{OD}_{\mathrm{BSA}}=$ the optical density with only BSA in the plate.

SPT of recombinant TEF 2. SPTs were performed with purified and endotoxin-removed TEF $2(0.01 \mathrm{mg} / \mathrm{ml})$. dissolved in phosphate buffer (pH 7.4, $50 \mathrm{mM}$ phosphate buffer, $100 \mathrm{mM}$ $\mathrm{NaCl}$ ). Glycerin was added to a final concentration of $50 \%$. The sample contained histamine phosphate $(5 \mathrm{mg} / \mathrm{ml})$ as the positive control or saline as a negative control. The results were checked 20 min after the SPT. The results were classified as follows: The prick spot became a wheal and fleck surrounding the wheal, it was positive $(+) ; 3+$, the response was the same as or stronger that of the histamine control; $2+$, the response was weaker than that of the histamine control but stronger than that of the negative control; $1+$, the response was significantly weaker than that of the histamine control but slightly stronger than that of the negative control; negative, no response (21).

Analysis of immunogenicity by immunoblotting. SPTs and sera from non-allergic children were used to assess immunogenicity. TEF 2 was subjected to $12 \%$ SDS-PAGE and transferred to a nitrocellulose membrane for immunoblot analysis. The membrane was blocked with $3 \%$ BSA for $1 \mathrm{~h}$ at $37^{\circ} \mathrm{C}$ and incubated with SPTs and sera from non-allergic children (diluted 1:5 with 5\% BSA-PBST, PBST: PBS containing $0.05 \%$ Tween $20 ; 100 \mu \mathrm{l} /$ well) for $1 \mathrm{~h}$ at room temperature. After washing five times with TBST, the membrane was incubated with a secondary antibody (peroxidase-conjugated goat anti-human IgE, 1:2,000, A18793, Invitrogen; Thermo Fisher Scientific, Inc.) for $1 \mathrm{~h}$ at $37^{\circ} \mathrm{C}$. Finally, after washing three times with TBS-T, the membrane was developed with a DAB kit (Invitrogen; Thermo Fisher Scientific, Inc.).

Bioinformatics analysis of TEF 2. The open reading frame (ORF) of TEF 2 was analyzed using the NCBI database (http://www.ncbi.nlm.nih.gov/), its physicochemical properties were predicted using the ProtParam Tool (http://web. expasy.org/protparam/), and the amino acid sequence was predicted using the Translate Tool (http://web.expasy. org/translate/) $(22,23)$. NetPhos3.1 (http://www.cbs.dtu. $\mathrm{dk} /$ services/NetPhos/) was used to predict phosphorylation sites, and Subcellular localization of cathepsin D was predicted by CELLO 2.5 (24). ProtScale (http://web.expasy. org/protscale/) was used to assess the hydrophilicity, average flexibility and the relative mutability $(25,26)$. Secondary structural elements were obtained using DNAStar. InterPro5.0 (http://www.ebi.ac.uk/interpro/), ScanProsite (http://prosite. expasy.org/scanprosite/) and MotifScan (http://myhits.isb-sib. ch/cgi-bin/motif_scan) was used to assess functional sites. The phylogenetic tree was constructed using BLAST, MEGA 5.0 and ClustalX 2.1 software (27). The Bioinformatics Predicted Antigenic Peptides (BPAP) system (http://imed.med.ucm. es/Tools/antigenic.pl), DNAStar Protean system (DNAStar Inc, Madison, WI, USA) and the BepiPred 1.0 server (http://www. cbs.dtu.dk/services/BepiPred/) were used to predict the B cell epitopes of TEF 2 (28-30). Furthermore, the Immune Epitope Database, Propre, SYFPEITHI, Net-MHCII 2.2 server and NetMHCIIpan-3.0 server were used to study the T cell epitopes of Der f TEF 2 (28-30).

Statistical analysis. All data are presented as the mean \pm SD and analyzed SPSS 18.0 statistical software (SPSS, Inc.). Student's t-test was used for the mean differences between two groups. $\mathrm{P}<0.05$ was considered to indicate a statistically significant difference. 
A

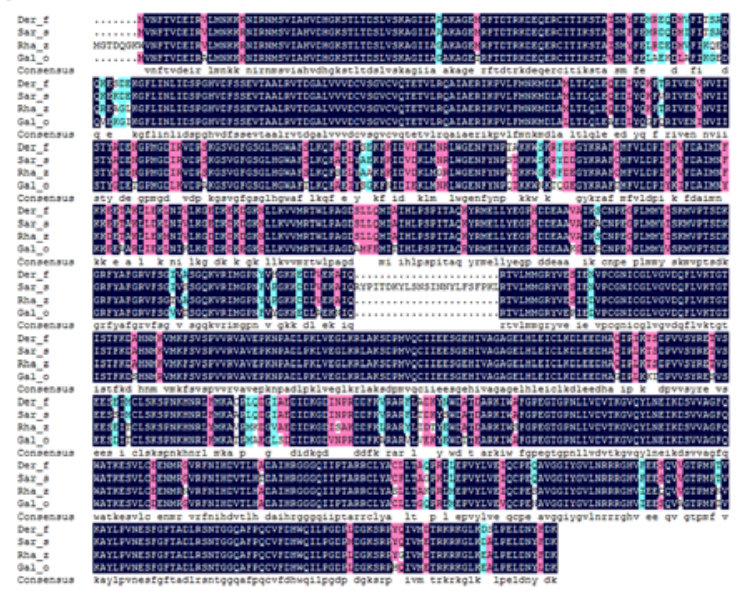

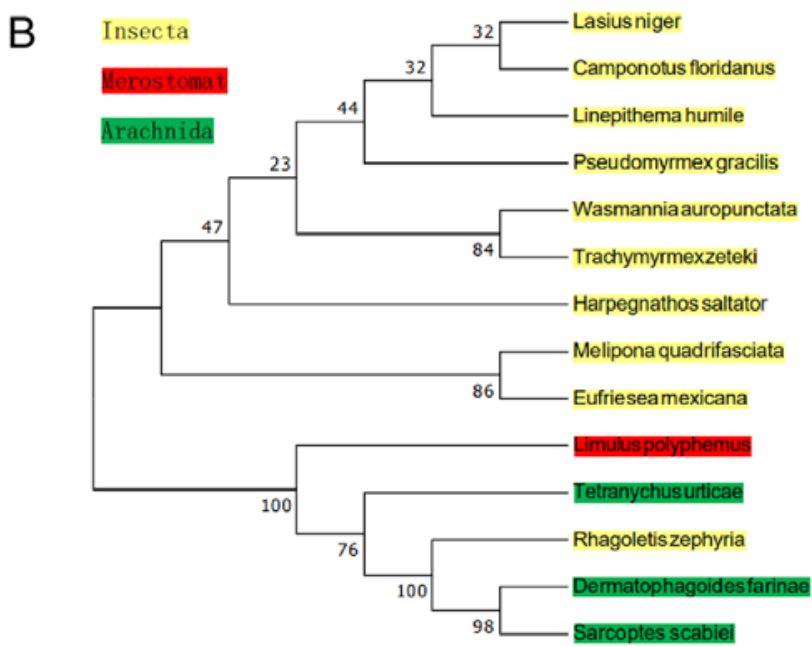

Figure 2. Analysis of the homologous alignment and phylogenetic relationship of Der f TEF 2. (A) Analysis of homologous alignment between Der $\mathrm{f}$ TEF 2 and other species. (B) Phylogenetic relationship of Der f TEF 2. Der f, Dermatophagoides farinae; TEF 2, translation elongation factor 2; Sar s, Sarcoptes scabiei; Rha z, Rhagoletis zephyria; Gal o, Galendromus occidentalis.

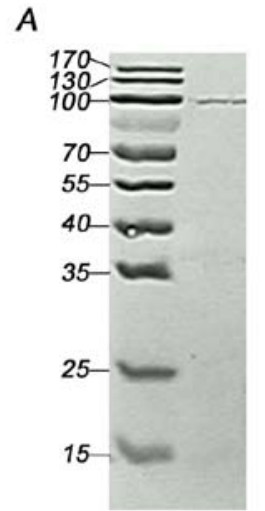

B

$C$

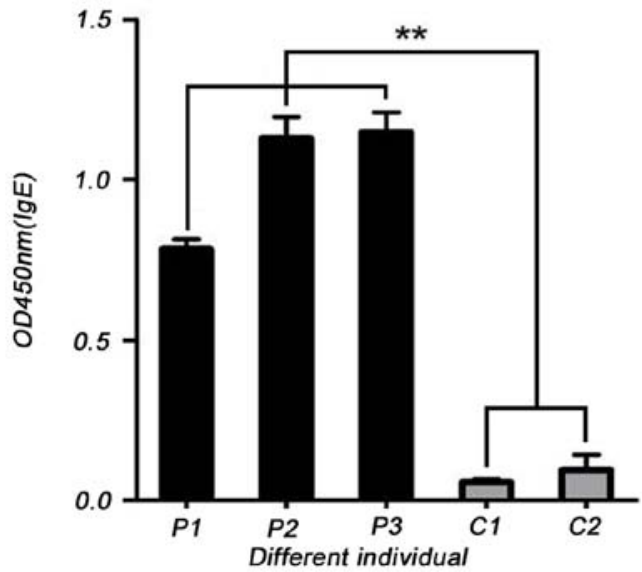

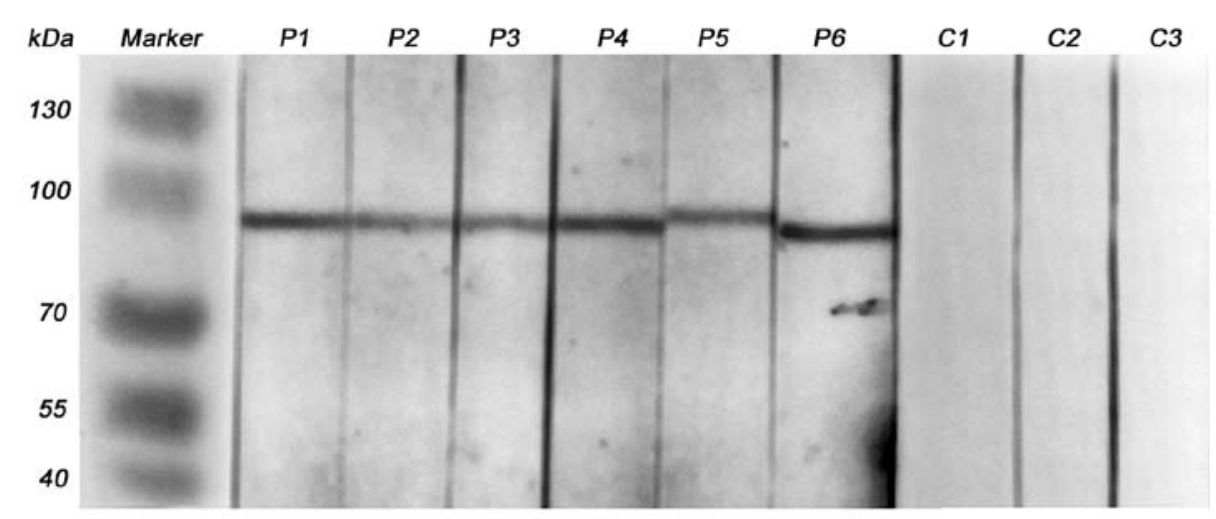

$D$

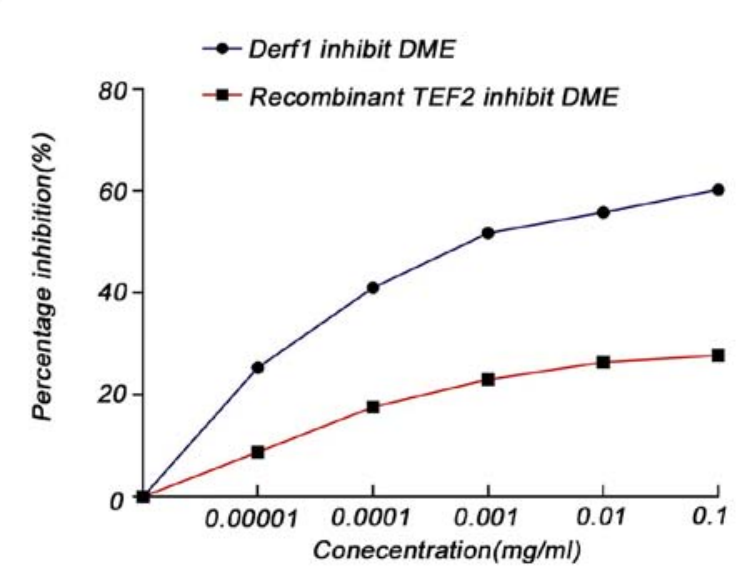

Figure 3. Immunogenicity of recombinant TEF 2. (A) The protein band indicates the purified Der f TEF 2, M: protein Maker (kDa); 1: The recombinant Der f TEF 2 purified by affinity chromatography. (B) Western blotting indicated that recombinant TEF 2 binds to IgE in the sera from patients allergic to house dust mites. Lanes: M, protein marker (kDa); 1, purified recombinant TEF 2. (C) Specific IgE reactivity to TEF 2 was determined using ELISAs. P1-P3, serum from TEF 2-positive patients; C1-C2; serum from healthy controls. (D) IgE binding to extracts was inhibited by recombinant TEF $2 .{ }^{* *} \mathrm{P}<0.01$. TEF 2 , translation elongation factor 2 .

\section{Results}

Synthesizing and analysis of amino acid sequence homology, alignment and molecular evolution. The cDNA of Der $\mathrm{fTEF} 2$ showed bright bands at $\sim 2,000$ bp after double enzymatic digestion (Fig. 1A). The sequencing results showed that one ORF of Der f TEF 2 is 2,535 bp long and encodes 844 amino acids from the ATG start codon to the TAA stop codon (Fig. 1B). Alignment between Der f TEF 2 and the homologous amino acid sequences from different species of Insecta, 
Table I. Results of skin prick tests.

\begin{tabular}{|c|c|c|c|c|c|c|}
\hline \multirow[b]{2}{*}{ Subject } & \multirow[b]{2}{*}{ Sex/age } & \multirow[b]{2}{*}{ Diagnosis } & \multicolumn{4}{|c|}{ Net wheal size $(\mathrm{mm})$, level } \\
\hline & & & DME & Histamine & PS & r-Der f TEF 2 \\
\hline 1 & Female/47 & $\mathrm{BA}, \mathrm{AR}, \mathrm{FA}$ & $4.5,++$ & 8 & 0 & 0 \\
\hline 2 & Female/37 & $\mathrm{AR}$ & $2.5,++$ & 5 & 0 & 0 \\
\hline 3 & Female/33 & & $1.5,+$ & 5.75 & 0 & 0 \\
\hline 4 & Male/24 & $\mathrm{BA}, \mathrm{AR}$ & $2,+$ & 5 & 0 & $1.5,+$ \\
\hline 5 & Male/33 & $\mathrm{BA}$ & $10,+++$ & 6.5 & 0 & 0 \\
\hline 6 & Male/36 & AR & $7.5,+++$ & 5 & 0 & 0 \\
\hline 7 & Female/43 & $\mathrm{BA}$ & $3.5,++$ & 5 & 0 & 0 \\
\hline 8 & Female/51 & $\mathrm{BA}, \mathrm{AR}$ & $2.5,++$ & 4.5 & 0 & 0 \\
\hline 9 & Female/18 & $\mathrm{BA}, \mathrm{AR}, \mathrm{DA}$ & $3.5,+++$ & 2 & 0 & 0 \\
\hline 10 & Female/42 & $\mathrm{BA}, \mathrm{AR}, \mathrm{FA}$ & $3.5,++$ & 5 & 0 & $2,+$ \\
\hline 11 & Female/47 & $\mathrm{BA}, \mathrm{AR}, \mathrm{FA}$ & $3,++$ & 6 & 0 & 0 \\
\hline 12 & Male/48 & $\mathrm{BA}, \mathrm{AR}$ & $5.5,+++$ & 4.5 & 0 & 0 \\
\hline 13 & Male/15 & $\mathrm{BA}, \mathrm{AR}$ & $6,++$ & 7.75 & 0 & 0 \\
\hline 14 & Female/39 & $\mathrm{AR}$ & $12.5,+++$ & 8.5 & 0 & 0 \\
\hline 15 & Male/63 & $\mathrm{BA}, \mathrm{DA}$ & $8.5,+++$ & 8 & 0 & 0 \\
\hline 16 & Male/15 & $\mathrm{BA}, \mathrm{AR}, \mathrm{FA}$ & $6.5,+++$ & 6 & 0 & 0 \\
\hline 17 & Male/12 & $\mathrm{BA}, \mathrm{AR}$ & $11,+++$ & 6 & 0 & 0 \\
\hline 18 & Female/18 & $\mathrm{BA}, \mathrm{AR}$ & $5.5,+++$ & 4.5 & 0 & 0 \\
\hline 19 & Female/25 & $\mathrm{AR}$ & $6.5,+++$ & 6.5 & 0 & 0 \\
\hline 20 & Female/40 & $\mathrm{BA}, \mathrm{AR}$ & $6.5,+++$ & 5 & 0 & 0 \\
\hline 21 & Male/75 & $\mathrm{BA}, \mathrm{AR}, \mathrm{FA}$ & $2.5,+$ & 6.5 & 0 & 0 \\
\hline 22 & Male/11 & $\mathrm{BA}, \mathrm{AR}, \mathrm{DA}$ & $6.5,+++$ & 5.5 & 0 & 0 \\
\hline 23 & Female/26 & $\mathrm{BA}$ & $1,+$ & 5.5 & 0 & 0 \\
\hline 24 & Male/15 & $\mathrm{BA}, \mathrm{AR}, \mathrm{FA}$ & $7,++$ & 8 & 0 & $2,+$ \\
\hline 25 & Female/14 & $\mathrm{BA}, \mathrm{AR}$ & $6.5,+++$ & 6.5 & 0 & 0 \\
\hline 26 & Male/8 & $\mathrm{BA}, \mathrm{AR}, \mathrm{FA}$ & $1,+$ & 4.5 & 0 & 0 \\
\hline 27 & Female/43 & $\mathrm{BA}$ & $2,+$ & 4.5 & 0 & 0 \\
\hline 28 & Male/47 & $\mathrm{BA}, \mathrm{AR}$ & $3.5,++$ & 5.5 & 0 & 0 \\
\hline 29 & Male/45 & $\mathrm{AR}$ & $2,+$ & 7.5 & 0 & 0 \\
\hline 30 & Female/52 & $\mathrm{AR}, \mathrm{BA}$ & $1.5,+$ & 5.5 & 0 & 0 \\
\hline 31 & Male/20 & $\mathrm{BA}$ & $1.5,+$ & 5 & 0 & 0 \\
\hline 32 & Female/20 & BA & $2,+$ & 4 & 0 & 0 \\
\hline 33 & Female/13 & $\mathrm{AR}$ & $1.25,+++$ & 6 & 0 & $2,+$ \\
\hline 34 & Female/86 & $\mathrm{BA}, \mathrm{AR}$ & $9,+++$ & 5.5 & 0 & $2.75,++$ \\
\hline 35 & Female/64 & $\mathrm{BA}$ & $2.25,+$ & 6 & 0 & 0 \\
\hline 36 & Female/41 & $\mathrm{BA}, \mathrm{AR}$ & $3.5,++$ & 5.5 & 0 & 0 \\
\hline 37 & Female/44 & $\mathrm{BA}, \mathrm{AR}$ & $4.5,+++$ & 4.5 & 0 & $2,+$ \\
\hline
\end{tabular}

Positive (+), $\geq 1$; Negative, 0 . Level (+) indicates the relative fleck size compared with the histamine positive and PS negative controls: +++ , the response was the same as or stronger than that of the histamine control; ++ , the response was weaker than that of the histamine control but stronger than that of the negative control; +, the response was significantly weaker than that of the histamine control but slightly stronger than that of the negative control. R-Der f TEF 2, recombinant translation elongation factor 2 from Dermatophagoides farinae; BA, bronchial asthma; AR, allergic rhinitis; DA, drug allergy; MA, mango allergy; FA, food allergy; DME, dust mite extract; PS, physiological saline.

Merostomata and Arachnida was conducted using BLAST. Der f TEF 2 is 94, 93 and $87 \%$ similar to Sarcoptes scabiei (GenBank: KPM11996.1), Rhagoletis zephyria (GenBank: XP_017490130.1) and Galendromus occidentalis (GenBank: XP_003744110.1), respectively, indicating high homology
(Fig. 2A). The homologous sequence was output in FASTA format after using MEGA 5.0 and ClustalX 2.1 software to construct the molecular evolutionary tree. The results showed that the TEF 2 gene from Der f has a relatively close relationship with the TEF 2 gene from Lasius niger, 
A

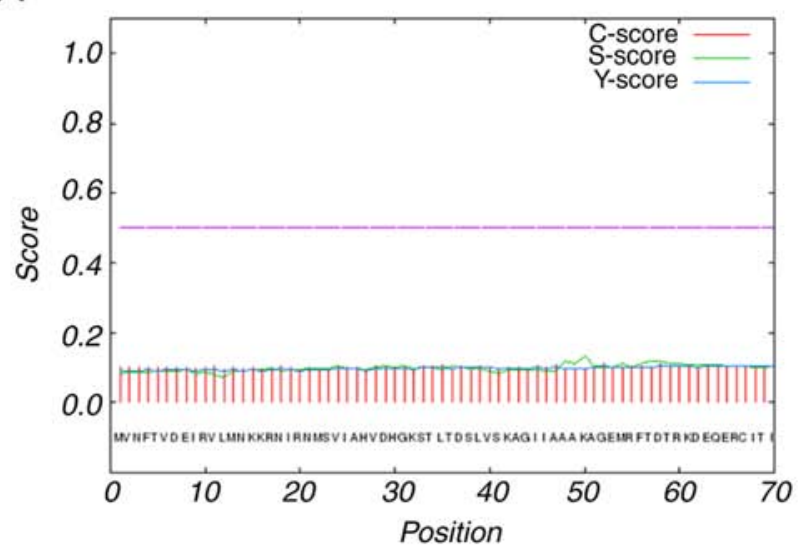

C

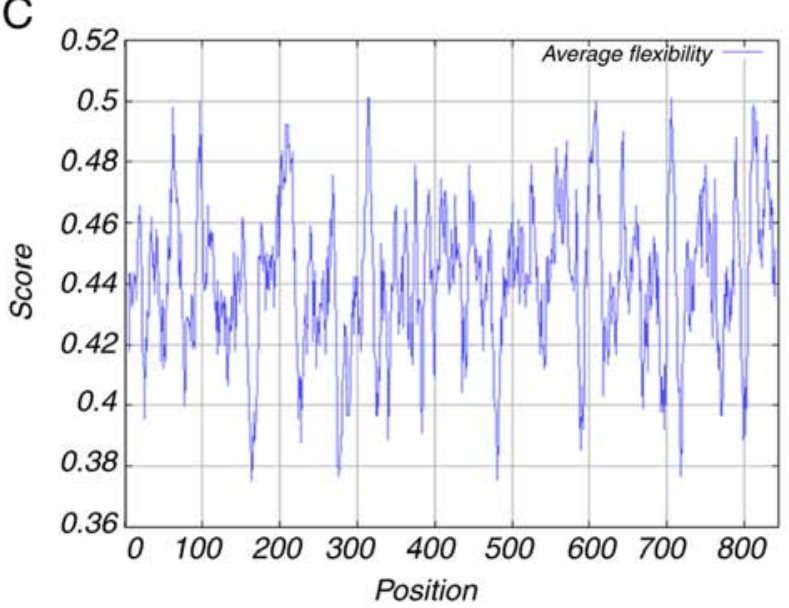

$\mathrm{E}$

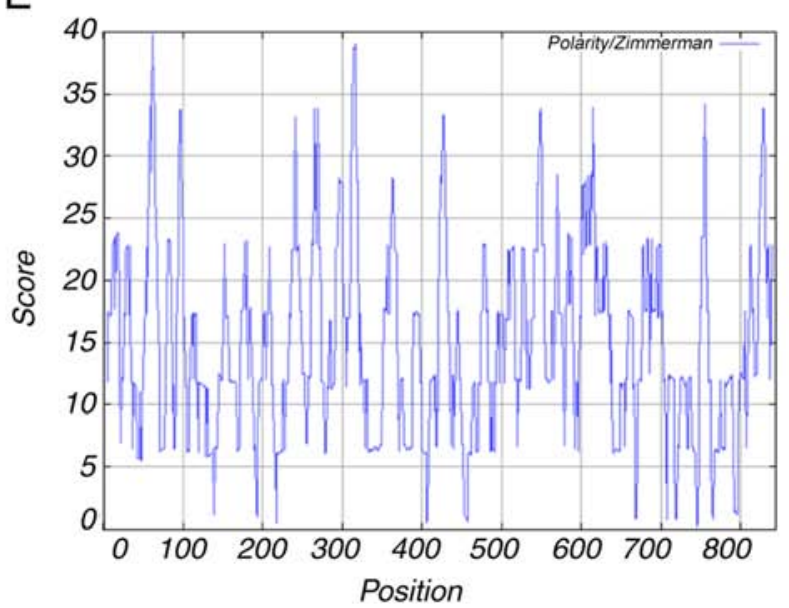

B

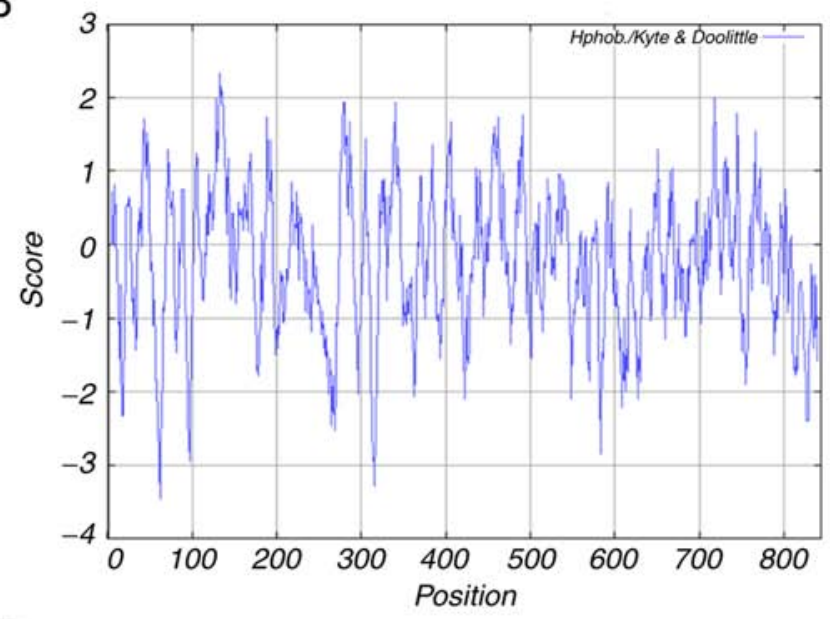

D

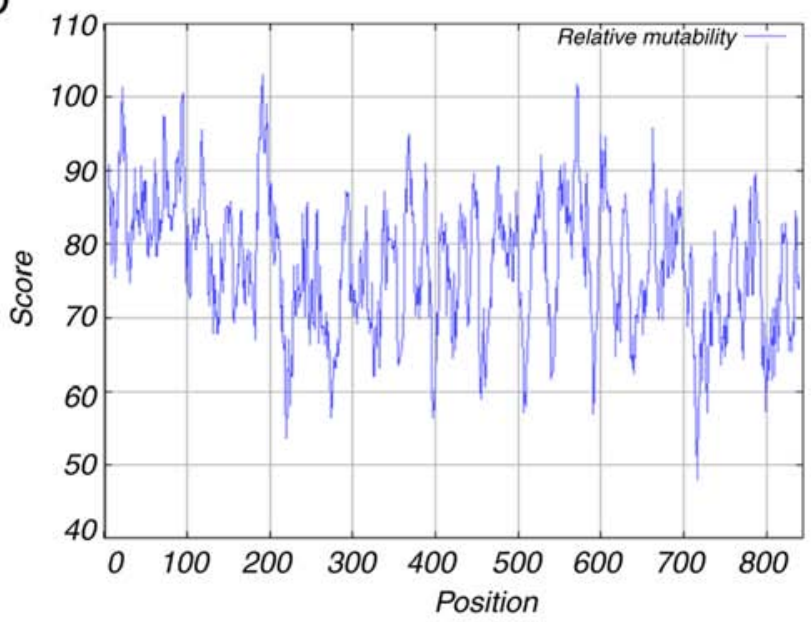

$\mathrm{F}$

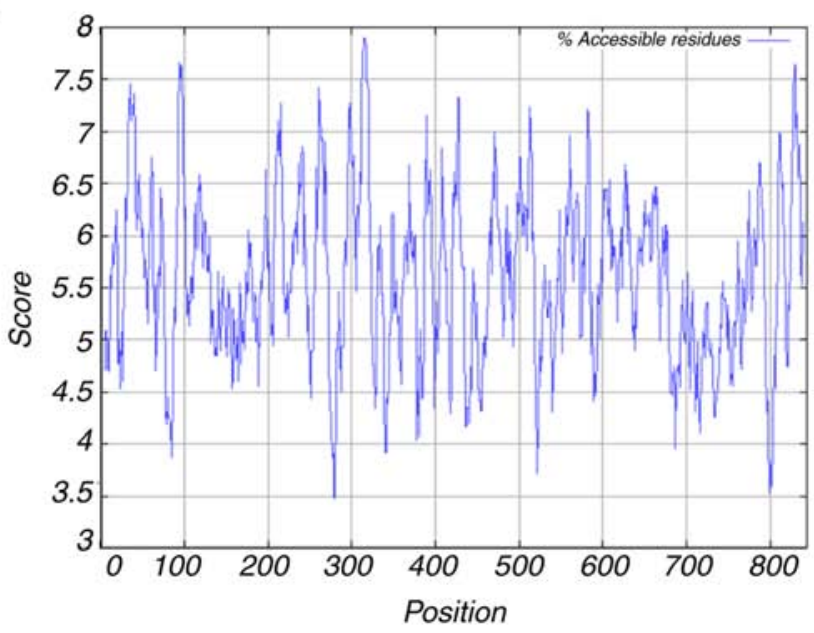

Figure 4. Bioinformatics analysis using the predicted amino acid sequence of Der $\mathrm{f}$ TEF 2. The $\mathrm{x}$-axis represents protein length from N-to C-terminal. The $\mathrm{y}$-axis represents the score, with a higher score indicating higher probability. (A) Prediction of signal peptide cleavage sites. (B) Hydrophilic analysis of Der f TEF 2 protein. (C) Average flexibility of Der f TEF 2. (D) Relative mutability of Der f TEF 2 (E) Polarity/Zimmerman score of Der f TEF 2. (F) Accessible residues of Der f TEF2. Der f, Dermatophagoides farinae; TEF 2, translation elongation factor 2.

D. farina, Camponotus floridanus, Harpegnathos saltator, Melipona quadrifasciata and Sarcoptes scabiei (Fig. 2B).

Immunogenicity of recombinant TEF 2. The SDS-PAGE analysis showed that TEF 2 was successfully expressed had a molecular weight of $\sim 100 \mathrm{kDa}$ as predicted (Fig. 3A). To determine the allergenicity of recombinant TEF 2 , immunoblot analysis was used to determine if the recombinant protein could react with serum from patients that are allergic to HDMs; the IgE-binding band had a molecular weight of $\sim 100 \mathrm{kDa}$ as predicted (Fig. 3B). The serum IgE from patients allergic to HDMs that was bound by recombinant TEF 2 was $>6$-fold higher compared with the serum IgE from healthy controls when quantified via ELISA (Fig. 3C). For ELISA 
Table II. Protein subcellular localization.

\begin{tabular}{llc}
\hline Support vector machine & Localization & Reliability \\
\hline Amino Acid compartment & Cytoplasmic & 0.973 \\
N-peptide compartment & Cytoplasmic & 0.704 \\
Partitioned seq. compartment & Cytoplasmic & 0.854 \\
Physico-chemical compartment & Cytoplasmic & 0.373 \\
Neighboring seq. compartment & Cytoplasmic & 0.942 \\
CELLO Prediction: & & \\
& Cytoplasmic & 3.847 \\
& Inner membrane & 0.580 \\
& Periplasmic & 0.442 \\
& Outer membrane & 0.088 \\
& Extracellular & 0.044 \\
\hline
\end{tabular}

inhibition assays, dilutions of recombinant TEF 2 or DME were incubated with the serum. The incubation inhibited the IgE binding of the serum obtained from patients to the coated DME or recombinant TEF 2 in a dose-dependent manner. The inhibition rate of recombinant TEF 2 against DME was $\sim 60 \%$ at $0.1 \mathrm{mg} / \mathrm{ml}$ (Fig. 3D). These results suggested that TEF 2 may be a novel allergen from the HDM allergen family that leads to type I hypersensitivity.

Clinical SPTs. SPTs were performed with Der f TEF2 in 37 patients sensitized to HDMs; 6 patients (16.2\%) showed a positive reaction (Table I).

Structural and functional prediction. As shown, there was no cleavage site in the amino acid sequence as predicted using SignalP 4.1 (Fig. 4A). Prediction of the physicochemical properties of Der f TEF 2 using the ProtParam Tool estimated that the molecular formula was $\mathrm{C}_{4,216} \mathrm{H}_{6,703} \mathrm{~N}_{1,141} \mathrm{O}_{1,240} \mathrm{~S}_{47}$, with a molecular weight of 94,722.32 Da and a theoretical isoelectric point of 6.25. The grand average of hydrophobicity (GRAVY) was predicted to be -0.222 , the aliphatic index was 87.86 and the instability index was predicted to be 34.13 . According to the definition provided by ProtParam, when the instability coefficient is $<40$, the protein is predicted to be stable; therefore, the TEF 2 protein was classified as stable. Combined with the analysis from the ProtScale software scale 'Hphob./Kyte $\&$ Doolittle', these results indicated the hydrophilicity of the protein. It showed that the most hydrophilic site of Der f TEF 2 appeared at amino acid position 62 with a score of -3.3456 , and the most hydrophobic site appeared at amino acid position 132 with a score of 2.333 (Fig. 4B). The maximum score for the average flexibility was calculated to be 0.501 at amino acid position 706; the minimum score at amino acid position 481 was 0.376 (Fig. 4C). The relative mutability was calculated to have a maximum score of 103.000 at amino acid position 191; the minimum score, at amino acid position 716 , was 47.889 (Fig. 4D). Using the Polarity/Zimmerman scale, the individual values were scored at a maximum of 39.766 at residue 62 and at a minimum of 0.237 at residue 745 (Fig. 4E). The accessible residues $(\%)$ were calculated as a minimum score of 3.478 at residues 279 and 280, and a maximum score of 7.900 at residues 314-316 (Fig. 4F).
NetPhos3.1 identified 31 phosphorylation sites. In total, 17 Ser residues $(23,41,76,97,109,116,211,214,264,391,404$, 488, 564, 570, 573, 664 and 833), $8 \mathrm{Thr}$ residues (59, 69, 298, $390,473,558,568$, and 824$)$ and 6 Tyr residues $(179,271$, $359,420,443$ and 840) were identified as potential phosphorylation sites (Fig. 5). The secondary structure prediction of Der f TEF 2 with DNAStar predicted $27 \alpha$-helices and $6 \beta$-sheets in the protein (Fig. 5). The subcellular localization of Der $f$ TEF 2 was predicted to be in the plasma membrane and extracellular space (Table II). Moreover, Der f TEF 2 was predicted to be a P-loop-containing nucleoside triphosphate hydrolase, Translation protein family member with $\mathrm{G}_{-} \mathrm{TR} \_2$ and G_TR_1 peptidase domains (Table III).

BPAP, DNAStar Protean and BepiPred 1.0 were used to predict the B cell epitopes of Der f TEF 2. The hydrophilicity, surface accessibility and flexibility of proteins play an important role in the formation of antigens. When the hydrophilic score is $>0$, the antigen index is $>0$ and surface accessibility is $>1$, an epitope is likely to be formed. Consolidating the predictions of these three tools, 17 potential $\mathrm{B}$ cell epitopes were identified on Der f TEF 2 (amino acids 29-35, 55-64, 92-99, 173-200, 259-272, 311-318, 360-365, 388-395, 422-428, 496-502, 512-518, 567-572, 580-586, 602-617, 785-790, 811-817 and 827-836; Fig. 5). The SYFPEITHI, NetMHCII 2.2 server, NetMHCIIpan-3.0 server, Immune Epitope Database and Propred were applied to predict the $\mathrm{T}$ cell epitopes of Der $\mathrm{f}$ TEF 2. Based on the results of these five immunoinformatics tools, the potential epitopes of Der f TEF 2 were predicted to comprise 14 peptide sequences (amino acids 1-15, 65-79, 120-134, 144-159, 236-250, 275-289, 404-418, 426-440, 463-477, 510-524, 644-658, 684-698, 716-730 and 816-830; Table IV and Fig. 5).

\section{Discussion}

The present study used high-throughput sequencing analysis of the HDM genome and transcriptome to study an HDM gene that may be involved in the allergic response (20). Previous studies have shown that HDM allergens are diverse, and numerous mite allergens have not been completely identified or characterized (31). The identification and characterization of novel HDM allergens may promote the development of improved diagnostic tools and immunotherapeutic vaccines. TEF 2 is one of the most abundant proteins in eukaryotes and plays an important role in various cellular processes, such as the regulation of cellular energy metabolism and apoptosis $(32,33)$. It has been reported that Rubber Elongation Factor is the major allergen in latex gloves, and triggers occupational latex allergy in an increasing number of healthcare workers and patients (34). The genes of Der f TEF 2 are highly conserved among different species, indicating that there is cross-reactivity for this protein. Thus, it was important to investigate the allergenicity of TEF 2 in D.farinae.

In the present study, Der f TEF 2 was identified and characterized as a novel allergen from $D$. farinae via cloning and expression of the recombinant protein. The recombinant Der f TEF 2 is encoded by a 2,535-bp ORF that produces a predicted sequence of 844 amino acids. Using a SPT, Der f TEF 2 was shown to be an important allergen and was found to react with the sera from $16.2 \%$ of patients sensitized to HDMs. Furthermore, it was found 


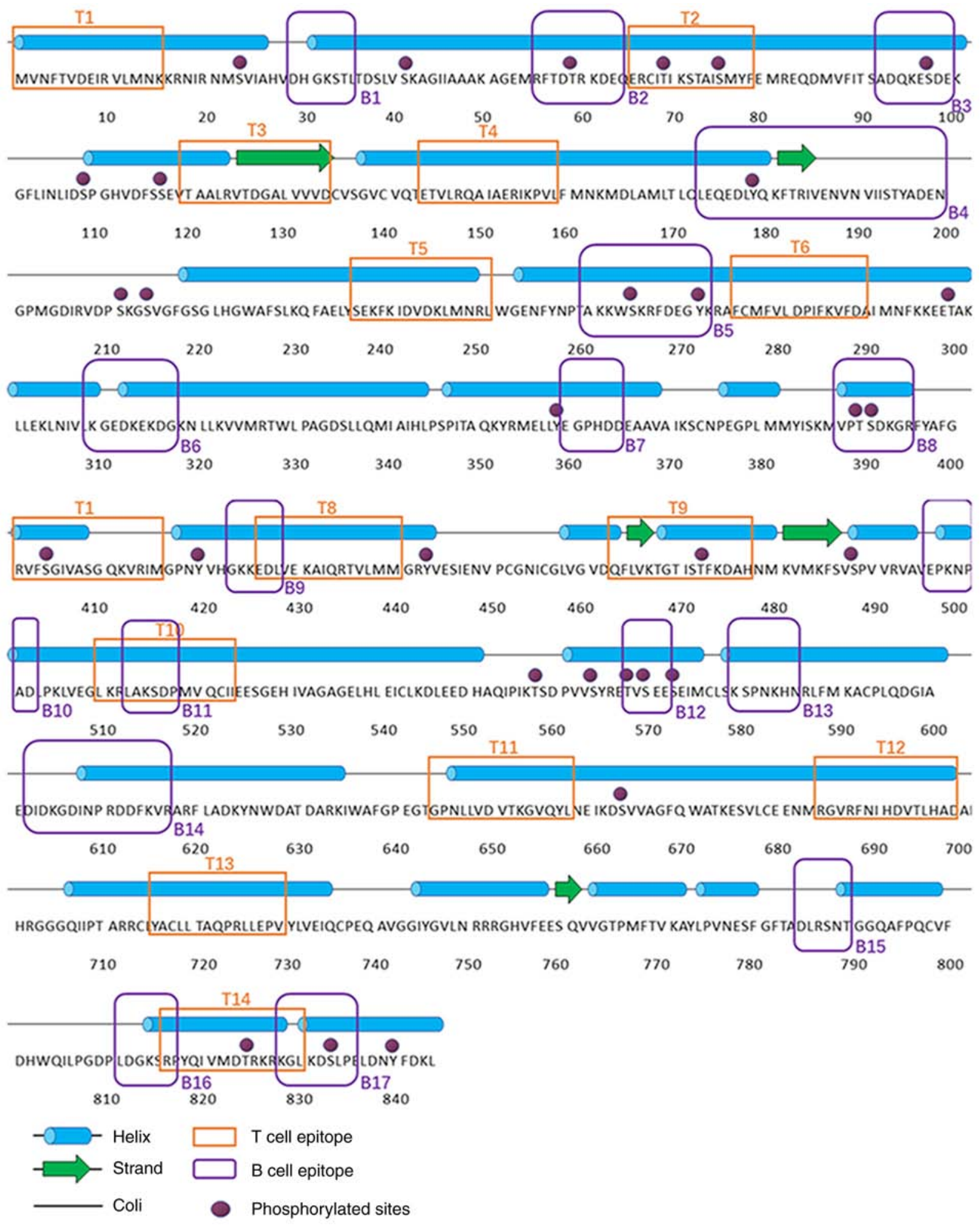

Figure 5. Sequence and secondary structure analysis of the Der $\mathrm{f}$ TEF 2 allergen. In total, $27 \alpha$-helices and $6 \beta$-sheets were identified in Der $\mathrm{f}$ TEF 2 . B cell epitopes, T cell epitopes and phosphorylation sites were also indicated. Der f, Dermatophagoides farinae; TEF 2, translation elongation factor 2; T, T cell epitope; B, B cell epitope.

that the serum IgE from patients allergic to HDMs bound to the recombinant TEF 2 protein and that the 6 patients with positive SPT results showed high levels of recombinant TEF2-specific IgE in the sera, as determined by ELISA and immunoblotting. Immune inhibition assays showed IgE cross-reactivity between recombinant Der f TEF 2 and DME. These results suggested that Der $\mathrm{f}$ TEF 2 is a novel type of allergen from HDMs.
Bioinformatics is an important technique for predicting the corresponding sequences, structures, functional properties and allergenicity of proteins (35). In the present study, the amino acid physicochemical parameters, homology, phosphorylation sites, secondary structure, and the $\mathrm{T}$ cell and B cell epitopes of Der f TEF 2 were analyzed via bioinformatics tools. The predicted results showed that 
Table III. Functional sites or motifs in the allergen.

Prediction tool

Functional sites or motifs

Amino acid position

InterPro 5.0

P-loop containing nucleoside triphosphate hydrolase

4-345

Small GTP-binding protein domain

20-174

Translation protein, $\beta$-barrel domain

$346-483$

Translation elongation factor EFTu-like, domain 2

$396-471$

EFG domain III/V-like

486-561

Ribosomal protein S5 domain 2-type fold

$563-727$

Ribosomal protein S5 domain 2-type fold, subgroup

$580-720$

Translation elongation factor EFG/EF2, domain IV

608-723

Elongation factor EFG, domain V-like

$725-812$

Tr-type $\mathrm{G}$ domain, conserved site

$58-73$

ScanProsite

Tr-type G domain profile(G_TR_2)

17-348

Tr-type $\mathrm{G}$ domain signature $\left(\mathrm{G}_{-} \mathrm{TR} \_1\right)$

$58-73$

EFTu, elongation factor thermo unstable; G domain, guanine nucleotide-binding domain; tr-type, translational-type.

Table IV. Epitope prediction.

\begin{tabular}{|c|c|c|c|c|c|c|}
\hline Epitope, amino acid & Sequence & SYFPEITHI & NetMHCII & NetMHCIIpan & IEDB & Preprod \\
\hline $1-15$ & MVNFTVDEIRVLMNK & ++ & ++ & ++ & ++ & ++ \\
\hline $65-79$ & ERCITIKSTAISMYF & ++ & ++ & ++ & ++ & ++ \\
\hline $120-134$ & TAALRVTDGALVVVD & ++ & ++ & ++ & ++ & ++ \\
\hline $144-159$ & ETVLRQAIAERIKPVL & ++ & ++ & ++ & ++ & ++ \\
\hline $236-250$ & EKFKIDVDKLMNRL & ++ & ++ & ++ & ++ & ++ \\
\hline $275-289$ & FCMFVLDPIFKVFDA & ++ & ++ & ++ & ++ & ++ \\
\hline $404-418$ & SGIVASGQKVRIMGP & ++ & ++ & ++ & ++ & ++ \\
\hline $426-440$ & EDLVEKAIQRTVLMM & ++ & ++ & ++ & ++ & ++ \\
\hline $463-477$ & QFLVKTGTISTFKDA & ++ & ++ & ++ & ++ & ++ \\
\hline $510-524$ & LKRLAKSDPMVQCII & ++ & ++ & ++ & ++ & ++ \\
\hline $644-658$ & GPNLLVDVTKGVQYL & ++ & ++ & ++ & ++ & ++ \\
\hline 684-698 & RGVRFNIHDVTLHAD & ++ & ++ & ++ & ++ & ++ \\
\hline $716-730$ & ACLLTAQPRLLEPV & ++ & ++ & ++ & ++ & ++ \\
\hline $816-830$ & RPYQIVMDTRKRKGL & ++ & ++ & ++ & ++ & ++ \\
\hline
\end{tabular}

' ++ ' means the final predicting regions of T cell epitopes in the Epitope prediction.

Der f TEF 2 is highly homologous to various insect species, contains 17 serine, 8 threonine and 6 tyrosine residues that are potential phosphorylation sites, and is a relatively stable protein. Using the software DNAStar to predict the secondary structure of TEF 2, it was found that there are $27 \alpha$-helices and $5 \beta$-sheets folds within the protein. In addition to these indices, the average flexibility, relative mutability, polarity and the accessible residues were determined using ProtScale tools. These bioinformatic characterizations will facilitate the understanding of the relationship between the structure and function of HDM allergens. ProtParam predicted that the GRAVY score of Der f TEF 2 was -0.222 and the ProtScale prediction showed that the hydrophilic peptide chains were distributed throughout the amino acid sequence, and that there were significantly more hydrophilic than hydrophobic peptide chains. These tools showed that Der f TEF 2 is hydrophilic and soluble. The relative mutability indicates the probability that an amino acid is replaced by another amino acid. The relative mutability of Der $f$ TEF 2 was found to have the highest score at residue 716 . The accessible residues are the sum of the accessibility of the atoms, which, for Der $f$ TEF 2, were predicted with maximum scores at residues 314, 315 and 316; thus, the active site is located on the surface of the protein.

Antigenic epitopes, also known as antigenic determinants, are specific antigenic sites that can be recognized by the corresponding antibody or antigen receptor and are the main chemical substances of immune system recognition (36). 
According to the reverse vaccine method, the use of bioinformatics technology for $\mathrm{T}$ cell epitope prediction, and then effective immunological experiments to verify the bioinformatics results, not only ensures the accuracy of the results, but also can save the time and expense of generating synthetic peptides (37).

To improve the accuracy of the predicted results, three different tools were used (BPAP, DNAStar Protean and BepiPred 1.0) to synthesize the data and identify the B cell epitopes of Der f TEF 2 (amino acids 29-35, 55-64, 92-99, 173-200, 259-272, 311-318, 360-365, 388-395, 422-428, 496-502, 512-518, 567-572, 580-586, 602-617, 785-790, 811-817 and 827-836). According to a previous study, asthma is a disease associated with genetic inheritance and the environment and HDM-induced asthma was associated with the HLA-DRB1*0301 and HLA-DRB1*0401 alleles (38). Therefore, the alleles HLA-DRB1*0301 and HLA-DRB $1 * 0401$ were used to predict the $\mathrm{T}$ cell epitopes of the D. farinae allergen TEF 2. Based on the results of five algorithms (SYFPEITHI, NetMHCII 2.2 server, NetMHCIIpan-3.0, IEDB and Propred), 14 HLA-DRB1*0301and HLA-DRB1*0401-restricted candidate T cell epitopes of Der f TEF 2 antigens were obtained (amino acid 1-15, 65-79, 120-134, 144-159, 236-250, 275-289, 404-418, 426-440, 463-477, 510-524, 644-658, 684-698, 716-730 and 816-830). The use of biotechnology to obtain recombinant allergens can provide novel insight and strategies for the development of specific immunotherapies and the treatment of allergic diseases.

In summary, the present study showed that TEF 2 exhibits high immunogenicity. The preset study also analyzed the structure and function of TEF 2 using bioinformatic technology, which may provide safe and sensitive reagents for the diagnosis and treatment of allergic diseases.

\section{Acknowledgements}

Not applicable.

\section{Funding}

The present study was supported by grants from the Natural Science Foundation of China (grant nos. 91442118 and 91542104), the Science and Technology Project of Guangdong Province (grant nos. 2013B031800023, 2014B090901041 and 2016A020216029), the Shenzhen Scientific Technology Basic Research Projects (grant nos. JCYJ20150525092941036 and JCYJ20160328144536436) and the PhD Start-up Fund of the Natural Science Foundation of Guangdong Province, China (grant no. 2014A030310192).

\section{Availability of data and materials}

The datasets used and/or analyzed during the current study are available from the corresponding author on reasonable request.

\section{Authors' contributions}

DC designed and performed experiments, analyzed data, interpreted the results and was a major contributor in writing the manuscript. QF and JL designed and performed experiments, analyzed data, interpreted the results and wrote the manuscript. $\mathrm{CH}, \mathrm{NH}, \mathrm{KXC}$ and $\mathrm{BS}$ provided key material and interpreted the results. ZL interpreted the results, supervised the study and edited the manuscript. All authors read and approved the final manuscript.

\section{Ethics approval and consent to participate}

Approval to conduct the present studies was obtained from the Ethic Committee of the Institutional Review Board of the School of Medicine, Shenzhen University. All participants, including children approved by legal guardians, provided written informed consent to participate in the present study.

\section{Patient consent for publication}

Not applicable.

\section{Competing interests}

The authors declare that they have no competing interests.

\section{References}

1. Arlian LG: House-dust-mite allergens: A review. Exp Appl Acarol 10: 167-186, 1991

2. Pawankar R, Canonica G and Holgate S: World allergy organization (WAO) white book on allergy. Update, 2013.

3. Valenta R, Linhart B, Swoboda I and Niederberger V: Recombinant allergens for allergen-specific immunotherapy: 10 years anniversary of immunotherapy with recombinant allergens. Allergy 66: 775-783, 2011.

4. Chapman MD, Smith AM, Vailes LD and Pomés A: Recombinant allergens for immunotherapy. Allergy Asthma Proc 23: 5-8, 2002 .

5. Bousquet $\mathbf{J}$ and Michel FB: Specific immunotherapy in asthma: Is it effective? J Allergy Clin Immunol 94: 1-11, 1994.

6. Thomas WR, Smith WA and Hales BJ: The allergenic specificities of the house dust mite. Chang Gung Med J 27: 563-569, 2004

7. Valenta R and Niederberger V: Recombinant allergens for immunotherapy. J Allergy Clin Immunol 119: 826-830, 2007.

8. Van Der Veen MJ, Jansen HM, Aalberse RC and van der Zee JS: Der p 1 and Der p 2 induce less severe late asthmatic responses than native Dermatophagoides pteronyssinus extract after a similar early asthmatic response. Clin Exp Allergy 31: 705-714, 2001.

9. Trombone AP, Tobias KR, Ferriani VP, Schuurman J, Aalberse RC, Smith AM, Chapman MD and Arruda LK: Use of a chimeric ELISA to investigate immunoglobulin $\mathrm{E}$ antibody responses to Der $\mathrm{p} 1$ and Der $\mathrm{p} 2$ in mite-allergic patients with asthma, wheezing and/or rhinitis. Clin Exp Allergy 32: 1323-1328, 2002.

10. Meyer CH, Bond JF, Chen MS and Kasaian MT: Comparison of the levels of the major allergens Der $p$ I and Der p II in standardized extracts of the house dust mite, Dermatophagoides pteronyssinus. Clin Exp Allergy 24: 1041-1048, 1994.

11. Shakib F, Ghaemmaghami AM and Sewell HF: The molecular basis of allergenicity. Trends Immunol 29: 633-642, 2008.

12. Chen DX, Zhou PK and Ran PX: Screening and identification of dominant allergens of Dermatophagoides pteronyssinus and Dermatophagoides farina. China Trop Med 39: 431-436, 2009.

13. Walker C, Muniz MF, Rolim JM, Martins RR, Rosenthal VC, Maciel CG, Mezzomo R and Reiniger LR: Morphological and molecular characterization of Cladosporium cladosporioides species complex causing pecan tree leaf spot. Genet Mol Res 15: $15,2016$.

14. Berthelot K, Lecomte S, Estevez Y, Coulary-Salin B, Bentaleb A, Cullin C, Deffieux A and Peruch F: Rubber elongation factor (REF), a major allergen component in Hevea brasiliensis latex has amyloid properties. PLoS One 7: e48065, 2012.

15. Chen R and Snyder M: Promise of personalized omics to precision medicine. Wiley Interdiscip Rev Syst Biol Med 5: 73-82, 2013. 
16. Bahcall O: Precision medicine. Nature 526: 335, 2015.

17. Ghaffari-Nazari H, Tavakkol-Afshari J, Jaafari MR, TahaghoghiHajghorbani S, Masoumi E and Jalali SA: Improving Multi-epitope long peptide vaccine potency by using a strategy that enhances CD $4^{+} \mathrm{T}$ help in BALB/c mice. PLoS One 10 e0142563, 2015 .

18. Nezafat N, Ghasemi Y, Javadi G, Khoshnoud MJ and Omidinia E: A novel multi-epitope peptide vaccine against cancer: An in silico approach. J Theor Biol 349: 121-134, 2014.

19. Shahsavandi S, Ebrahimi MM, Sadeghi K and Mahravani H: Design of a heterosubtypic epitope-based peptide vaccine fused with hemokinin-1 against influenza viruses. Virol Sin 30: 200-207, 2015

20. Chan TF, Ji KM, Yim AK, Liu XY, Zhou JW, Li RQ, Yang KY, Li J, Li M, Law PT, et al: The draft genome, transcriptome and microbiome of Dermatophagoides farinae reveal a broad spectrum of dust mite allergens. J Allergy Clin Immunol 135: 539-548, 2015

21. Lin JL, Wang YY, Xiao XJ, Wu YL, Sun BQ, Gao AJ, Liu ZG, $\mathrm{Li}$ J, Yang PC and Liu XY: Characterization of a new subtype of allergen in dermatophagoides farinae-Der $\mathrm{f} 28$. J Thorac Dis 7 : 1842-1849, 2015

22. Lafarga T, O'Connor $\mathrm{P}$ and Hayes M: Identification of novel dipeptidyl peptidase-IV and angiotensin-I-converting enzyme inhibitory peptides from meat proteins using in silico analysis. Peptides 59: 53-62, 2014.

23. Thirugnanasambantham K, Muralidaran S and Mandal AK: Molecular cloning, computational and expression analysis of anthocyanidin reductase in tea (Camellia sinensis). Appl Biochem Biotechnol 174: 130-145, 2014.

24. Yu CS, Lin CJ and Hwang JK: Predicting subcellular localization of proteins for Gram-negative bacteria by support vector machines based on n-peptide compositions. Protein Sci 13: 1402-1406, 2004.

25. Lu YJ, Lu G, Shi DZ, Li LH and Zhong SF: Bioinformatic analysis for structure and function of TCTP from Spirometra mansoni. Asian Pac J Trop Med 6: 709-712, 2013.

26. Seddigh S: Comprehensive comparison of two protein family of P-ATPases (13A1 and 13A3) in insects. Comput Biol Chem 68: 266-281, 2017.

27. Lin J, Wang H, Li M, Liang Z, Jiang C, Wu Y, Liu Z, Yang P and Liu X: Characterization and analysis of a cDNA coding for the group 29b (Der f 29b) allergen of Dermatophagoides farinae. Am J Transl Res 8: 568-577, 2016.

28. Chen H, Yang HW, Wei JF and Tao AL: In silico prediction of the T-cell and IgE-binding epitopes of Per a 6 and Bla g 6 allergens in cockroaches. Mol Med Rep 10: 2130-2136, 2014.
29. LiX, Yang HW, Chen H, Wu J,Liu Y and Wei JF: In silico prediction of T and B cell epitopes of Der $\mathrm{f} 25$ in Dermatophagoides farinae. Int J Genomics 2014: 483905, 2014.

30. Yang H, Chen H, Jin M, Xie H, He S and Wei JF: Molecular cloning, expression, IgE binding activities and in silico epitope prediction of Per a 9 allergens of the American cockroach. Int J Mol Med 38: 1795-1805, 2016.

31. An S, Chen L, Long C, Liu X, Xu X, Lu X, Rong M, Liu Z and Lai R: Dermatophagoides farinae allergens diversity identification by proteomics. Mol Cell Proteomics 12: 1818-1828, 2013.

32. Faller WJ, Jackson TJ, Knight JR, Ridgway RA, Jamieson T, Karim SA, Jones C, Radulescu S, Huels DJ, Myant KB, et al: mTORC1-mediated translational elongation limits intestinal tumour initiation and growth. Nature 517: 497-500, 2015.

33. Leprivier G, Remke M, Rotblat B, Dubuc A, Mateo AR, Kool M, Agnihotri S, El-Naggar A, Yu B, Somasekharan SP, et al: The eEF2 kinase confers resistance to nutrient deprivation by blocking translation elongation. Cell 153: 1064-1079, 2013.

34. Marchetti-Deschmann M and Allmaier G: Allergenic compounds on the inner and outer surfaces of natural latex gloves: MALDI mass spectrometry and imaging of proteinous allergens. J Mass Spectrom 44: 61-70, 2009.

35. Cui Y, Teng F, Yu L, Zhou Y, Zhang $\mathrm{C}$ and Yang L: Dermatophagoides farinae allergen Der f 9: Cloning, expression, purification, characterization and IgE-binding in children with atopic asthma. Pediatr Pulmonol 52: 282-292, 2017.

36. Yu S, Zhang H, Yao D, Liu W, Wang X, Chen X, Wei Y, Zhang Z, Wang J, Yu L, et al: Identification of CD4 ${ }^{+} \mathrm{T}$-cell epitopes on iron-regulated surface determinant B of Staphylococcus aureus. Microb Pathog 89: 108-113, 2015.

37. Masignani V, Rappuoli R and Pizza M: Reverse vaccinology: A genome-based approach for vaccine development. Expert Opin Biol Ther 2: 895-905, 2002.

38. Movahedi M, Moin M, Gharagozlou M, Aghamohammadi A, Dianat S, Moradi B, Nicknam MH, Nikbin B and Amirzargar A: Association of HLA class II alleles with childhood asthma and Total IgE levels. Iran J Allergy Asthma Immunol 7: 215-220, 2008.

This work is licensed under a Creative Commons Attribution-NonCommercial-NoDerivatives 4.0 International (CC BY-NC-ND 4.0) License. 